\title{
THEORY OF FOUR-POINT DIRECT-CURRENT POTENTIAL DROP MEASUREMENTS ON A METAL PLATE
}

\author{
Nicola Bowler
}

Center for Nondestructive Evaluation, lowa State University, Ames, lowa, USA

\begin{abstract}
Four-point direct-current potential drop techniques are well-suited for accurate, nondestructive measurement of material conductivity (or resistivity). From measurements of potential drop on a large metal plate, the electrical conductivity of the plate can be inferred if the thickness of the plate and the dimensions of the probe are known. In this work, an expression for the voltage measured by a four-point probe in contact with a large metal plate of arbitrary thickness is derived from an analytic series representation of the electric field in the plate. The position of the voltage pickup points is permitted to be offset with respect to the current injection points, allowing analysis of colinear, rectangular, and square-head probes. Analytical asymptotic expressions are derived in the limiting cases of plates that are thin or thick with respect to the dimensions of the probe. The range of plate thicknesses for which the asymptotic expressions are valid is determined as a function of relevant probe dimensions. The results of this study provide a useful guide in selecting the most appropriate arrangement of probe points for measurement of the electrical conductivity of large metal plates, depending on the level of accuracy required. Theory is compared with experimental data for measurements made with colinear probes on a variety of metal plates and very good agreement is obtained.
\end{abstract}

Keywords: Four-point probe, potential drop measurement, electrical conductivity measurement, metal plate

\section{INTRODUCTION}

There is a need for portable instruments that have the ability to measure material conductivity nondestructively, to high accuracy and preferably without the need for calibration standards. Applications include metal sorting and alloy identification [1], monitoring the heat treatment of aluminum alloys [2], and the detection of damage that gives rise to a change in material conductivity, $\sigma$, such as thermal damage in aircraft structures [3]. The conductivity of common metals varies by around two orders of magnitude. Copper is highly conductive and lends its name to the International Annealed Copper Standard (IACS), a measure of conductivity used to compare electrical conductors to a traditional copper-wire standard, in which $100 \%$

Address correspondence to Nicola Bowler, Center for Nondestructive Evaluation, Applied Sciences Complex 11, 1915 Scholl Road, Ames, IA 50011-3042, USA. E-mail: nbowler@iastate.edu 
IACS represents $\sigma=58 \mathrm{MS} / \mathrm{m}$. Titanium, for example, has $\sigma \approx 0.58 \mathrm{MS} / \mathrm{m}$ (Table 1). The conductivities of some other common metal alloys are also given in Table 1.

In a recent study [4], the uncertainties associated with measurement of electrical conductivity of metal plates using three different electromagnetic nondestructive measurement methods were compared. It was found that measurements made using a commercial eddy-current instrument (Zetec MIZ-21A) and a hand-built eddy-current probe coupled with an Agilent 4294A impedance analyzer, gave similar levels of uncertainty in $\sigma(\approx 2 \%$ for good conductors $)$ although the commercial instrument performed more poorly for low-conductivity metals such as stainless steel (for which $\sigma \approx 1 \mathrm{MS} / \mathrm{m}$ ). A four-point potential drop method performed best, with uncertainty approximately $0.5 \%$ for measurements on brass, stainless steel, and spring steel. A discussion of possible sources of error in eddy-current conductivity measurements is given in Ref. [5]. Factors such as variations in specimen geometry and temperature are important. In particular, $\sigma$ is strongly dependent on specimen temperature, whereas microstructural variations such as large grains and local dislocations in a metal alloy have a much less significant effect. For example, in recent work on characterization of shot-peened nickel-base superalloys, the change in $\sigma$ due to residual stresses is described as being small, typically less than $1 \%[6]$.

This article focuses on establishing the accuracy of various theoretical expressions for the voltage measured using the four-point direct-current potential drop (DCPD) technique, for different probe-plate configurations. The accuracy of these expressions directly reflects the accuracy with which $\sigma$ can be measured.

DCPD measurements have the advantage of being independent of the magnetic permeability of the metal, so that the technique can be used to measure the electrical conductivity of ferrous metals, whereas eddy-current methods cannot. The DCPD method also works well for low-conductivity materials such as semiconductors [7] and in geophysical applications $[8,9]$, because the measured potential is inversely proportional to $\sigma$ and,

TABLE 1 Metal Plate Alloys and Conductivity, $\sigma$, Thickness, $T$, and Lateral Dimensions, $w \times I$

\begin{tabular}{llcccc}
\hline Plate & \multicolumn{1}{c}{ Alloy } & $\sigma(\mathrm{MS} / \mathrm{m})$ & $T(\mathrm{~mm})$ & $w \times I(\mathrm{~mm})$ & Reference \\
\hline Brass & C26000 & $16.42 \pm 0.09$ & $5.66 \pm 0.01$ & $615 \times 616$ & {$[4]$} \\
Aluminum & 2024 & $17.6 \pm 0.2$ & $101 \pm 1$ & $149 \times 202$ & - \\
Titanium & Ti-6Al-4V & $0.58 \pm 0.01$ & $12.47 \pm 0.01$ & $318 \times 331$ & - \\
Stainless Steel & 316 & $1.379 \pm 0.007$ & $6.36 \pm 0.01$ & $457 \times 457$ & {$[4]$} \\
Spring Steel & C1074/75 & $5.50 \pm 0.04$ & $1.57 \pm 0.01$ & $412 \times 412$ & {$[4]$} \\
Carbon Steel & 1018 & $5.18 \pm 0.04$ & $6.30 \pm 0.02$ & $616 \times 619$ & {$[15]$} \\
\hline
\end{tabular}


therefore, the signal-to-noise ratio improves as $\sigma$ decreases. Eddy-current methods lose accuracy as $\sigma$ decreases.

In Ref. [4], potential drop measurements were made on plates whose thickness was significantly smaller than the length of the four-point probe, so that a simple analytic formula could be used to infer the value of $\sigma$ from the measured potential, $V$. In this article, the mathematical analysis is generalized to deal with plates of arbitrary thickness with respect to the probe dimensions for a variety of probe configurations. Initially, a series summation expression for $V$ is obtained by integrating, between the voltage pickup points, an analytic expression for the electric field in the plate. The position of the pickup points is permitted to be offset with respect to the current injection points, so that both colinear and rectangular arrangements of the probe points can be modeled. The plate is assumed sufficiently large that edge effects are negligible. Then, closed-form asymptotic expressions for the potential drop are derived in two regimes; for plates that are either thick or thin with respect to the dimensions of the probe. The accuracy of these asymptotic expressions is investigated as a function of plate thickness and probe dimensions, for both colinear and rectangular probes, by numerical comparison with results obtained using the series summation. Explicit expressions for $V$ are given for colinear, rectangular, and square arrangements of the probe points.

The results of this study provide a useful guide in selecting the most appropriate arrangement of probe points for measurement of the electrical conductivity of conductive plates, depending on the level of accuracy required in the measurement.

As a validation exercise, theory is compared with experimental data for measurements made with colinear probes on a variety of metal plates. Very good agreement is obtained.

\section{CALCULATION OF DCPD VOLTAGE}

Consider the four-point probe arrangement shown in Fig. 1. The DCPD voltage, $V$, measured between pickup points at $(p, c, 0)$ and $(q, c, 0)$, can be calculated using the formula

$$
V=-\int_{(p, c, 0)}^{(q, c, 0)} \mathbf{E} \cdot d \mathbf{l}
$$

where $\mathbf{E}$ denotes the electric field. Choosing the path of integration to be a straight line joining the pickup points allows Eq. (1) to be written as

$$
V=-\int_{p}^{q} E_{x}(x, c, 0) d x
$$




$\frac{\text { air }}{\text { conductive plate }} z=T$

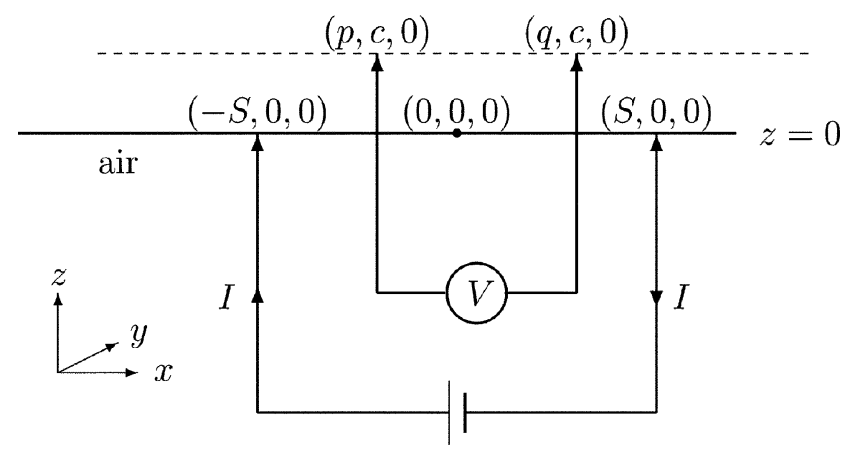

FIGURE 1. Four-point probe in contact with a conductive plate, thickness $T$. The current injection points are located at $(x, y, z)=( \pm S, 0,0)$. The voltage pickup points at $(p, c, 0)$ and $(q, c, 0)$ can occupy any plane of constant $y$. Here they are shown in the plane $y=c$. I represents the applied direct current.

The electric field in the plate can be expressed as the superposition of fields separately associated with each of the current injection/extraction points. For one current injection wire lying on the axis of a cylindrical coordinate system, the components of the electric field in the plate due to the injected current can be expressed in terms of the following series summations [10]:

$$
\begin{gathered}
E_{\rho}(\mathbf{r})=\frac{I \rho}{2 \pi \sigma} \sum_{n=0}^{\infty}\left[\frac{1}{r_{n}^{3}}+\frac{1}{\left(r_{n}^{\prime}\right)^{3}}\right] \\
E_{z}(\mathbf{r})=\frac{I}{2 \pi \sigma} \sum_{n=0}^{\infty}\left\{\frac{(z+2 n T)}{r_{n}^{3}}+\frac{[z-2(n+1) T]}{\left(r_{n}^{\prime}\right)^{3}}\right\}
\end{gathered}
$$

In Eqs. (3) and (4), $\rho\left(=\sqrt{x^{2}+y^{2}}\right)$ and $z$ are radial and axial coordinates, respectively, of a cylindrical coordinate system, centered on the current wire. I represents the applied direct current. $T$ and $\sigma$ represent the thickness and conductivity of the plate, respectively. The term $r_{n}=\sqrt{\rho^{2}+(z+2 n T)^{2}}$ and $r_{n}^{\prime}=\sqrt{\rho^{2}+[z-2(n+1) T]^{2}}$. The term containing $r_{0}$ is, by itself, the solution for a half-space conductor, in which the current density radiates 
uniformly from the point of injection and $J_{r}(\mathbf{r})=\sigma E_{r}(\mathbf{r})=I /\left(2 \pi r^{2}\right)$. Higher terms represent contributions to the current density due to internal reflections from the plate surfaces. These contributions act as though originating at image sources located at $(0,0, \pm 2 n T)$, with $n=1,2,3, \ldots$.

The component of the electric field required for evaluation of the integral in Eq. (2) can be expressed in terms of $E_{\rho}$ as follows:

$$
E_{x}(x, c, 0)=\frac{(x+S)}{\rho_{+}} E_{\rho}\left(\rho_{+}, 0\right)-\frac{(x-S)}{\rho_{-}} E_{\rho}\left(\rho_{-}, 0\right)
$$

in which $\rho_{ \pm}=\sqrt{(x \pm S)^{2}+c^{2}}$. Now insert $E_{X}$ as given in Eq. (5) into the integral of Eq. (2) and make the change of variable $X=x \pm S$. Then Eq. (2) can be written as the sum

$$
V=-\mathcal{I}_{+}+\mathcal{I}_{-}
$$

where

$$
\mathcal{I}_{ \pm}=\int_{p \pm S}^{q \pm S} \frac{X}{\sqrt{X^{2}+c^{2}}} E_{\rho}(X, c, 0) d X
$$

From Eq. (3),

$$
E_{\rho}(x, y, 0)=\frac{l}{2 \pi \sigma}\left\{\frac{1}{\rho^{2}}+2 \rho \sum_{n=1}^{N} \frac{1}{\left[\sqrt{\rho^{2}+(2 n T)^{2}}\right]^{3}}\right\}
$$

which gives, when substituted into Eq. (7),

$$
\mathcal{I}_{ \pm}=\frac{I}{2 \pi \sigma} \int_{p \pm S}^{q \pm S}\left\{\frac{X}{\left(X^{2}+c^{2}\right)^{3 / 2}}+2 \sum_{n=1}^{N} \frac{X}{\left[\sqrt{X^{2}+c^{2}+(2 n T)^{2}}\right]^{3}}\right\} d X
$$


Straightforward evaluation of the integrals in Eq. (9) and use of Eq. (6) gives

$$
\begin{aligned}
V=\frac{I}{2 \pi \sigma} \sum_{n=0}^{\infty} \epsilon_{n} & {\left[\frac{1}{\sqrt{(S+q)^{2}+c^{2}+(2 n T)^{2}}}-\frac{1}{\sqrt{(S-q)^{2}+c^{2}+(2 n T)^{2}}}\right.} \\
& \left.-\frac{1}{\sqrt{(S+p)^{2}+c^{2}+(2 n T)^{2}}}+\frac{1}{\sqrt{(S-p)^{2}+c^{2}+(2 n T)^{2}}}\right]
\end{aligned}
$$

where $\epsilon_{m}$ is the Neumann factor with properties $\epsilon_{0}=1$ and $\epsilon_{m}=2$, $m=1,2,3, \ldots$ The expression for $V$ given in Eq. (10) is valid for arbitrary plate thickness and for a probe whose pickup points may be offset by distance $c$ from the line of the current injection/extraction points.

Practically, the influence of the plate edges on the electromagnetic field may give rise to errors when Eq. (10) is applied. For a colinear probe with equal spacing between the four points, errors due to the proximity of the plate edges are calculated to be less than $1 \%$ if the plate dimensions are approximately four times greater than the probe length and the probe is placed centrally on the plate [1]. It has also been shown that errors are smaller for such a probe placed parallel to the shorter plate dimension $[1,11]$ and for probes whose pickup points are moved closer to the current injection points, rather than having the four points equally spaced [11].

There are two limiting regimes in which $V$ can be represented by simple analytic expressions. These correspond to plates that are either thick or thin with respect to the dimensions of the probe.

\subsection{Thick Plate Asymptote}

Taking the limit $T \rightarrow \infty$ in Eq. (10) yields the result for a half-space conductor, $V_{\infty}$. All terms in the series cancel except those for $n=0$, which leaves

$$
\begin{aligned}
V_{\infty}=\frac{I}{2 \pi \sigma}[ & \frac{1}{\sqrt{(S+q)^{2}+c^{2}}}-\frac{1}{\sqrt{(S-q)^{2}+c^{2}}}-\frac{1}{\sqrt{(S+p)^{2}+c^{2}}} \\
& \left.+\frac{1}{\sqrt{(S-p)^{2}+c^{2}}}\right]
\end{aligned}
$$


Putting $c=0$ in Eq. (11) yields a result that agrees with expressions given in Ref. [12] and Ref. [7], Section 1.2, for arbitrary separation of colinear probe points on a half-space conductor.

\subsection{Thin Plate Asymptote}

Following Uhlir [12], note the relationship

$$
M(\lambda)+N(\lambda)=\frac{1}{\lambda}+2 \ln \lambda-2 \ln 2+2 \gamma
$$

in which

$$
\begin{gathered}
M(\lambda)=2 \sum_{n=1}^{\infty}\left(\frac{1}{n}-\frac{1}{\sqrt{n^{2}+\lambda^{2}}}\right) \\
N(\lambda)=2 \pi \sum_{n=1}^{\infty} i H_{0}^{(1)}(i 2 \pi n \lambda)
\end{gathered}
$$

$i=\sqrt{-1}$ and $\gamma=0.577216 \ldots$ is Euler's constant [13, Eq. 4.1.32]. Now rewrite Eq. (10) in the form

$$
V=V_{\infty}+\frac{l}{2 \pi \sigma T} \sum_{n=1}^{\infty}\left(\frac{1}{\sqrt{n^{2}+Q_{+}^{2}}}-\frac{1}{\sqrt{n^{2}+Q_{-}^{2}}}-\frac{1}{\sqrt{n^{2}+P_{+}^{2}}}+\frac{1}{\sqrt{n^{2}+P_{-}^{2}}}\right)
$$

where $V_{\infty}$ is given in Eq. (11), $Q_{ \pm}=\left[\sqrt{(S \pm q)^{2}+C^{2}}\right] / 2 T$, and similarly for $P_{ \pm}$. Making use of Eqs. (12) and (13), Eq. (15) can be written

$$
\begin{aligned}
V= & -\frac{I}{2 \pi \sigma T}\left\{\ln \left[\frac{\sqrt{(S+q)^{2}+c^{2}}}{\sqrt{(S-q)^{2}+c^{2}}} \frac{\sqrt{(S-p)^{2}+c^{2}}}{\sqrt{(S+p)^{2}+c^{2}}}\right]\right. \\
& \left.-\frac{1}{2}\left[N\left(Q_{+}\right)-N\left(Q_{-}\right)-N\left(P_{+}\right)+N\left(P_{-}\right)\right]\right\}
\end{aligned}
$$


For large $|\lambda|, N(\lambda)$ can be written [13, Eq. 9.2.3],

$$
N(\lambda) \sim 2 \sum_{n=1}^{\infty} \frac{e^{-2 \pi n \lambda}}{\sqrt{n \lambda}}, \quad|\lambda| \longrightarrow \infty
$$

Hence, $N(\lambda) \longrightarrow 0$ as $\lambda \longrightarrow \infty$ or, $N\left(Q_{ \pm}\right)$and $N\left(P_{ \pm}\right) \longrightarrow 0$ as $T \longrightarrow 0$. From Eq. (16) this leaves the following expression valid in a thin plate regime, whose range of validity is yet to be determined:

$$
V_{\mathrm{TP}}=-\frac{I}{2 \pi \sigma T} \ln \left[\frac{\sqrt{(S+q)^{2}+c^{2}}}{\sqrt{(S-q)^{2}+c^{2}}} \frac{\sqrt{(S-p)^{2}+c^{2}}}{\sqrt{(S+p)^{2}+C^{2}}}\right]
$$

\section{EXAMPLES}

In this section, two special cases concerning probe configurations in common usage are examined in detail.

\subsection{Colinear, Symmetric Probe}

In a colinear four-point probe, all four points are arranged on the same straight line. Typically the pickup points are placed symmetrically with respect to the current injection/extraction points. With these restrictions, $c=0$ and $p=-q$. Also truncating the infinite series to $N+1$ terms, Eq. (10) reduces to

$$
V^{\mathrm{LS}}=\frac{I}{\pi \sigma} \sum_{n=0}^{N} \epsilon_{n}\left[\frac{1}{\sqrt{(S+q)^{2}+(2 n T)^{2}}}-\frac{1}{\sqrt{(S-q)^{2}+(2 n T)^{2}}}\right]
$$

In Fig. 2, a family of curves is plotted, of $\pi \sigma V^{L S} S / I$ versus $T / S$, for various values of the ratio of pickup length to current injection length, $q / S$. From the figure it is clear that the measured voltage increases with increasing pickup length $q$ and decreasing plate thickness $T$. When the plate thickness is greater than approximately half of the probe length, that is $T \approx S$ or greater, $V^{L S}$ holds a constant asymptotic value.

3.1.1. Thick Plate Asymptote Taking the limit $T \longrightarrow \infty$ in Eq. (19), or putting $c=0$ and $p=-q$ in Eq. (11), yields the result for a half-space conductor, $V_{\infty}^{L S}$ :

$$
V_{\infty}^{\mathrm{LS}}=\frac{I}{\pi \sigma}\left[\frac{1}{(S+q)}-\frac{1}{(S-q)}\right]
$$




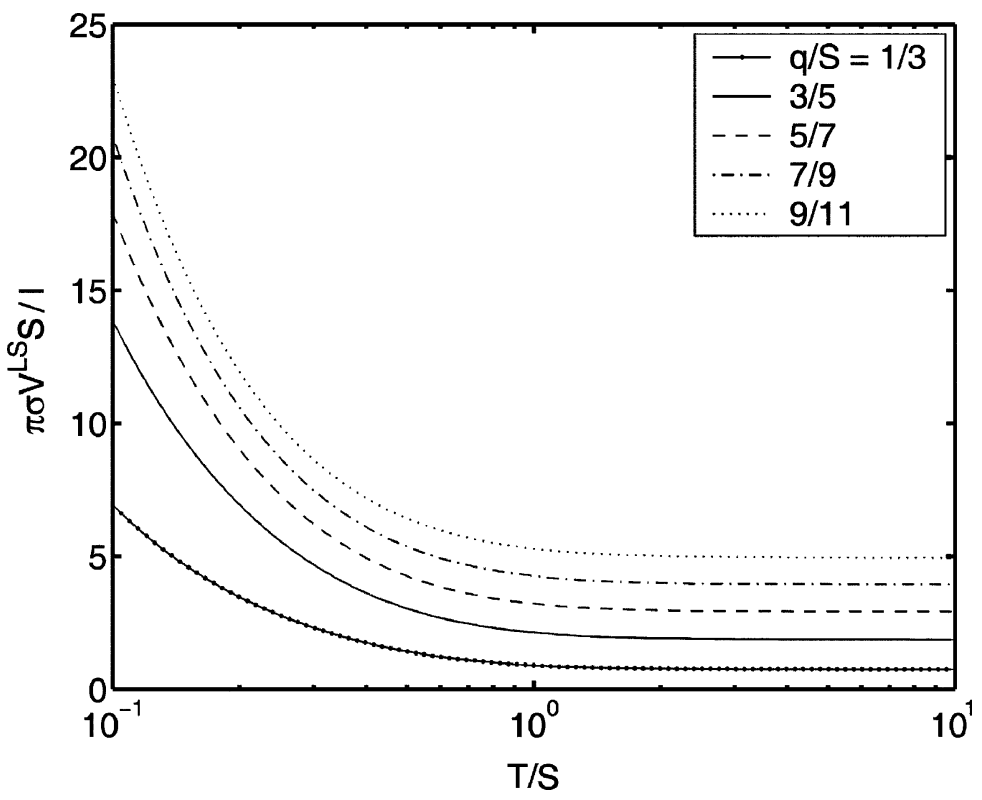

FIGURE 2. Dimensionless pickup voltage, $\pi \sigma V^{L S} S / I$, in the case of a colinear, symmetric probe, computed from Eq. (19) with $N=1000$, for $q / S=1 / 3(0.333), 3 / 5(0.600), 5 / 7(0.714), 7 / 9(0.778)$, and 9/11 (0.818).

For a probe in which the spacing between all four points is equal, then $S-q=a$, say, and $2 q=a$. The expression for $V_{\infty}^{\mathrm{LS}}$ reduces to

$$
V_{\infty}^{\mathrm{LE}}=-\frac{a}{2 \pi \sigma a}
$$

in agreement with the results given in Ref. [7, Section 1.2] and Ref. [14, Chap. 13, Part 5].

3.1.2. Thin Plate Asymptote Following the procedure of Section 2.2, or by putting $c=0$ and $p=-q$ in Eq. (16), Eq. (19) may be written in the form

$$
V^{\mathrm{LS}}=-\frac{I}{\pi \sigma T}\left\{\ln \left(\frac{S+q}{S-q}\right)-\frac{1}{2}\left[N\left(\lambda_{+}\right)-N\left(\lambda_{-}\right)\right]\right\}
$$

in which $\lambda_{ \pm}=(S \pm q) / 2 T$. In the thin plate regime (whose range of validity is yet to be determined),

$$
V_{\mathrm{TP}}^{\mathrm{LS}}=-\frac{I}{\pi \sigma T} \ln \left(\frac{S+q}{S-q}\right)
$$


3.1.3. Validity of Asymptotic Formulae In Fig. 3, curves of $\pi \sigma V^{L S} S / I$ versus $T / S$ are plotted showing the half-space asymptote, Eq. (20), the thin plate asymptote, Eq. (23), and the result valid for arbitrary plate thickness, Eq. (19). In this case the probe points are equally spaced; $q / S=1 / 3$. From Fig. 3 it can be seen that the thin plate asymptotic formula is a good approximation for $T \approx 0.3 S$ or smaller, whereas that for the thick plate works well for $T \approx 3 S$ or greater, for equally spaced probe points. The accuracy of these approximations will now be quantified through numerical comparison with $\checkmark$ given by Eq. (19) with $N=1000$.

The percentage difference

$$
\frac{V^{\mathrm{LS}}-V_{i}^{\mathrm{LS}}}{V^{\mathrm{LS}}} \times 100
$$

is plotted in Figs. 4 and 5 for $i=\infty$ and TP, respectively, for various values of $q / S$. It is clear from visual inspection of the curves in Figs. 4 and 5 that the accuracy of the asymptotes for any particular plate thickness also depends on the ratio of the pickup probe separation to the separation of the current input probes, $q / S$. The thick plate asymptotic expression becomes more accurate, for a given plate thickness, as the pickup length increases, that is, as $q / S$

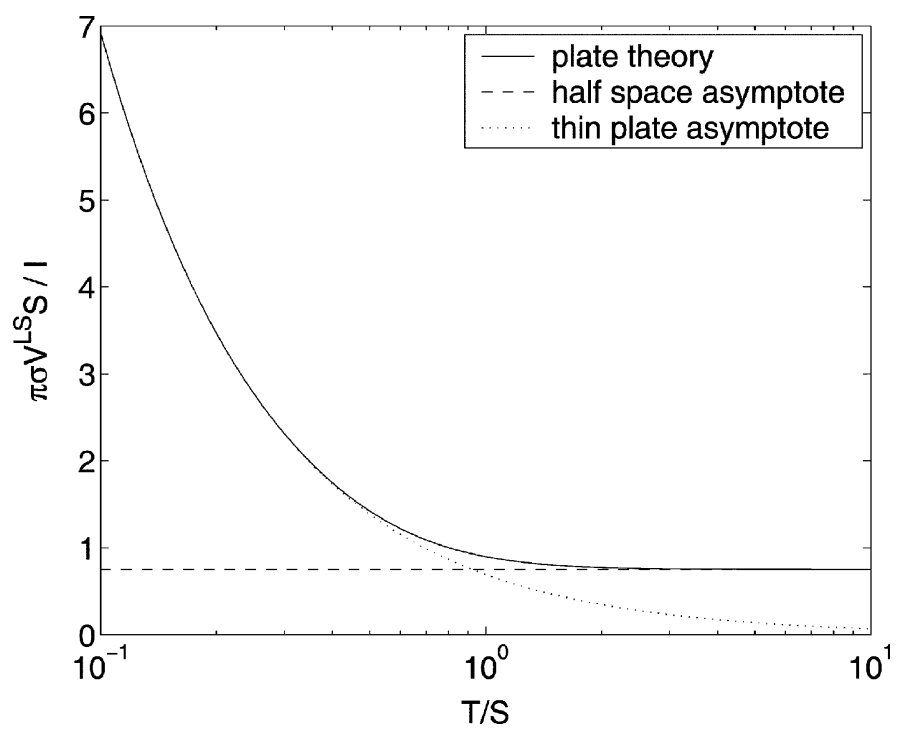

FIGURE 3. Dimensionless pickup voltage, $\pi \sigma V^{L S} S / I$, in the case of a colinear, symmetric probe $(q / S=1 / 3)$, computed from Eq. (19) with $N=1000$, plotted as a function of $T / S$. Thin and thick plate asymptotes computed from Eqs. (20) and (23), respectively, are shown. 


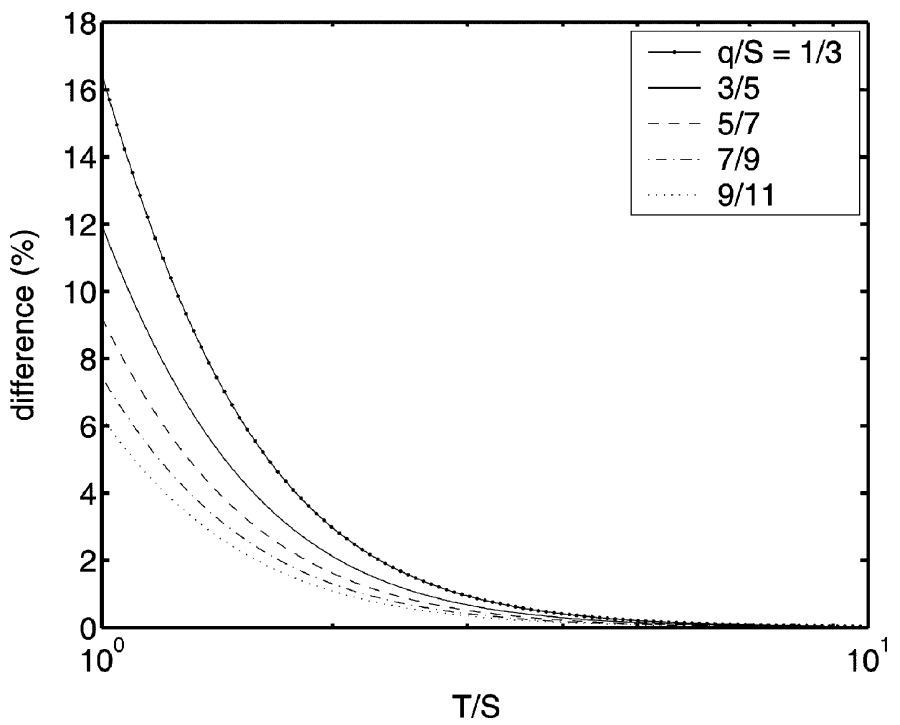

FIGURE 4. Percentage difference between pickup voltages calculated using Eq. (19) and thick plate asymptote Eq. (20), in the case of a colinear, symmetric probe, as a function of $T / S$, for various values of $q / S$.

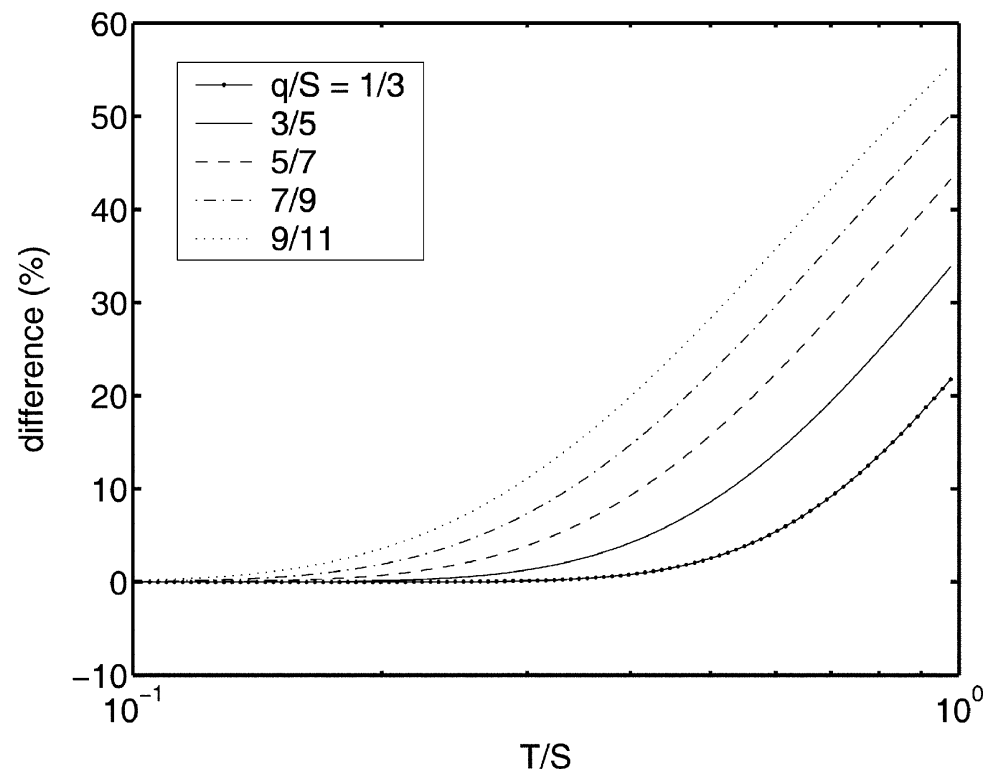

FIGURE 5. As for Fig. 4 but for thin plate asymptote Eq. (23). 
increases and the pickup points approach the current input points more closely. The thin plate asymptotic expression becomes more accurate in the opposite case, as the pickup points move away from the current input points ( $q / S$ decreases).

In Figs. 6 and 7 , curves of constant percentage difference $(0.1,1,5$, and $10 \%$ ) are plotted for $T / S$ versus $q / S$, for thick and thin plate asymptotes respectively. These curves facilitate proper choice of probe dimensions relative to plate thickness for the level of accuracy required in utilizing asymptotic Eqs. (20) or (23).

3.1.4. Comparison between Theory and Experimental Data In Fig. 8, theory is compared with experimental DCPD measurements made on various metal plates with three different linear, symmetric probes with $q / S=$ $0.240,0.399$, and 0.873. Some of the data is taken from Ref. [4] and [15], in which details of the experimental procedure can be found. Parameters of the metal plates and probes are given in Tables 1 and 2, respectively. The measured DCPD voltage, $V_{\text {exp }}^{L S}$, for various probe and plate combinations is tabulated (in terms of impedance, $V_{\exp }^{\mathrm{LS}} / I$ ) in Table 3 and plotted in Fig. 8. There is generally excellent agreement between theory and experimental data. The small discrepancy between theory and experiment for the point with $T / S=5.02$ (on aluminum) is attributed to edge effects.

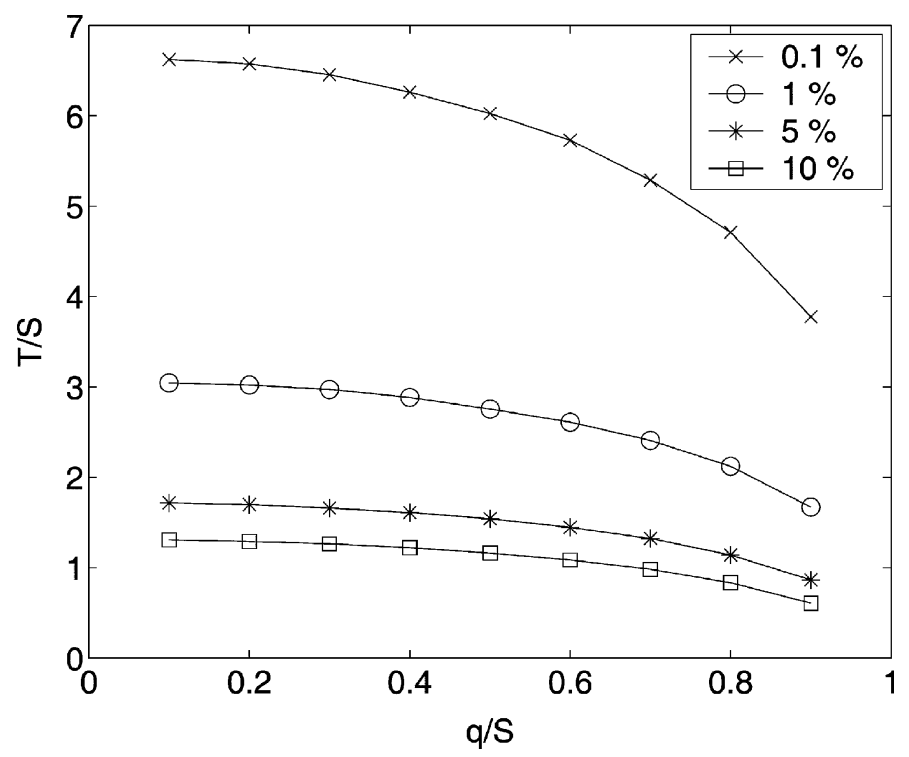

FIGURE 6. Curves of constant percentage difference between pickup voltages calculated using Eq. (19) and thick plate asymptote Eq. (20), in the case of a colinear, symmetric probe, for $T / S$ plotted versus $q / S$. 


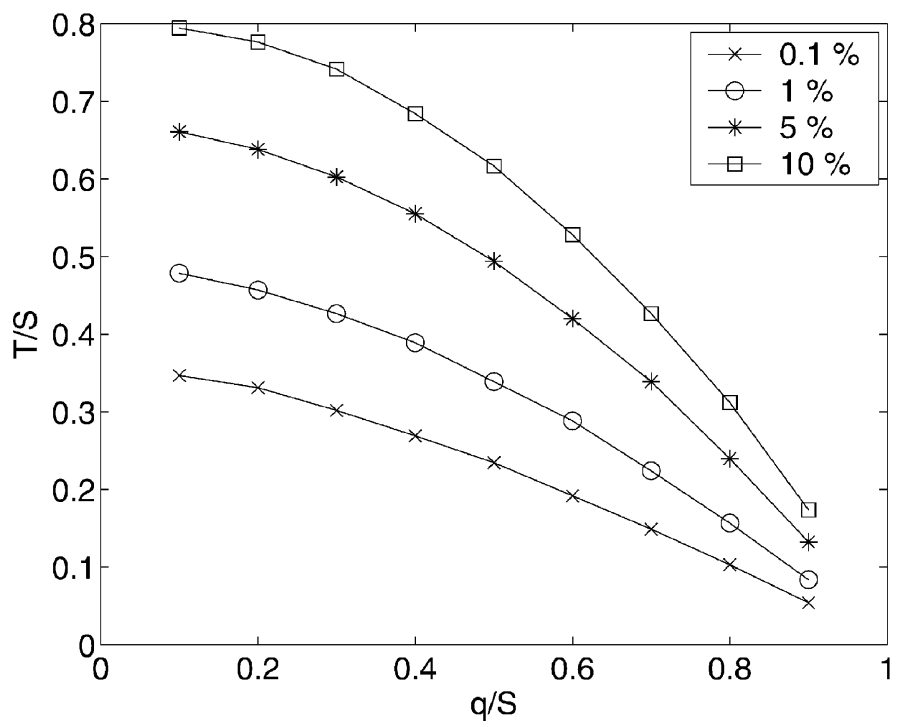

FIGURE 7. As for Fig. 6 but with thin plate asymptote Eq. (23).

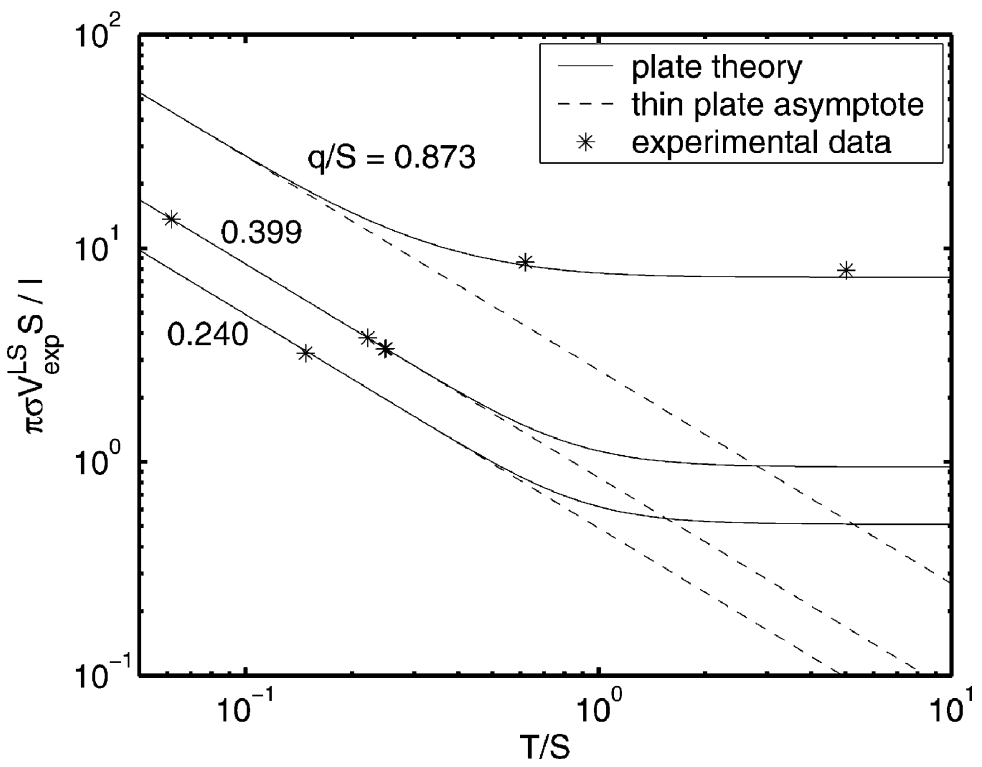

FIGURE 8. Theory and experimental data measured on various metal plates using three different colinear, symmetric probes. Plate and probe parameters are given in Tables 1-3. 
TABLE 2 Probe Half-Length, $S$, and Half-Pickup-Length, $q$ (see Fig. 1)

\begin{tabular}{lcc}
\hline Probe & $S(\mathrm{~mm})$ & $q(\mathrm{~mm})$ \\
\hline 1 & $25.454 \pm 0.005$ & $10.157 \pm 0.005$ \\
2 & $38.2 \pm 0.3$ & $9.18 \pm 0.01$ \\
3 & $20.105 \pm 0.005$ & $17.543 \pm 0.005$ \\
\hline
\end{tabular}

\subsection{Rectangular and Square Probes}

In a rectangular four-point probe, the pickup points form one side of the rectangle and the current injection/extraction points form the opposite side. With these restrictions, $p=-S$ and $q=S$, and Eq. (10) reduces to

$$
V^{\mathrm{R}}=\frac{I}{\pi \sigma} \sum_{n=0}^{N} \epsilon_{n}\left[\frac{1}{\sqrt{(2 S)^{2}+c^{2}+(2 n T)^{2}}}-\frac{1}{\sqrt{c^{2}+(2 n T)^{2}}}\right]
$$

In the case of a probe with points arranged on the vertices of a square, $c=2 S$ and Eq. (25) reduces further to

$$
V^{S}=\frac{I}{\pi \sigma} \sum_{n=0}^{N} \epsilon_{n}\left[\frac{1}{\sqrt{2 c^{2}+(2 n T)^{2}}}-\frac{1}{\sqrt{c^{2}+(2 n T)^{2}}}\right]
$$

In Fig. 9, a family of curves is plotted, of $\pi \sigma V^{R} S / I$ versus $T / S$, for various values of the ratio of offset length to current injection length, $c /(2 S)$. From the figure it is clear that the measured voltage increases with decreasing offset length $c$-as the pickup points approach more closely to the current injection points and the aspect ratio of the probe consequently increases-and as the plate thickness $T$ decreases.

TABLE 3 Measured DCPD Impedance, $V_{\exp }^{\mathrm{LS}} / I$, and Normalized Plate and Probe Dimensions

\begin{tabular}{lcccccc}
\hline Plate & Probe & $T / S$ & $q / S$ & $V_{\exp }^{\mathrm{LS}} / I(\mu \Omega)$ & Reference & $\pi \sigma V_{\exp }^{\mathrm{LS}} S / I$ \\
\hline Spring Steel & 1 & 0.0617 & 0.399 & $31.2 \pm 0.2$ & {$[4]$} & $13.7 \pm 0.1$ \\
Brass & 2 & 0.148 & 0.240 & $1.64 \pm 0.01$ & {$[15]$} & $3.23 \pm 0.03$ \\
Brass & 1 & 0.222 & 0.399 & $2.90 \pm 0.02$ & {$[4]$} & $3.81 \pm 0.03$ \\
Carbon Steel & 1 & 0.248 & 0.399 & $8.14 \pm 0.06$ & {$[15]$} & $3.37 \pm 0.04$ \\
Stainless Steel & 1 & 0.250 & 0.399 & $30.9 \pm 0.2$ & {$[4]$} & $3.39 \pm 0.03$ \\
Titanium & 3 & 0.62 & 0.873 & $235.8 \pm 0.5$ & - & $8.6 \pm 0.1$ \\
Aluminum & 3 & 5.02 & 0.873 & $7.13 \pm 0.04$ & - & $7.9 \pm 0.1$ \\
\hline
\end{tabular}




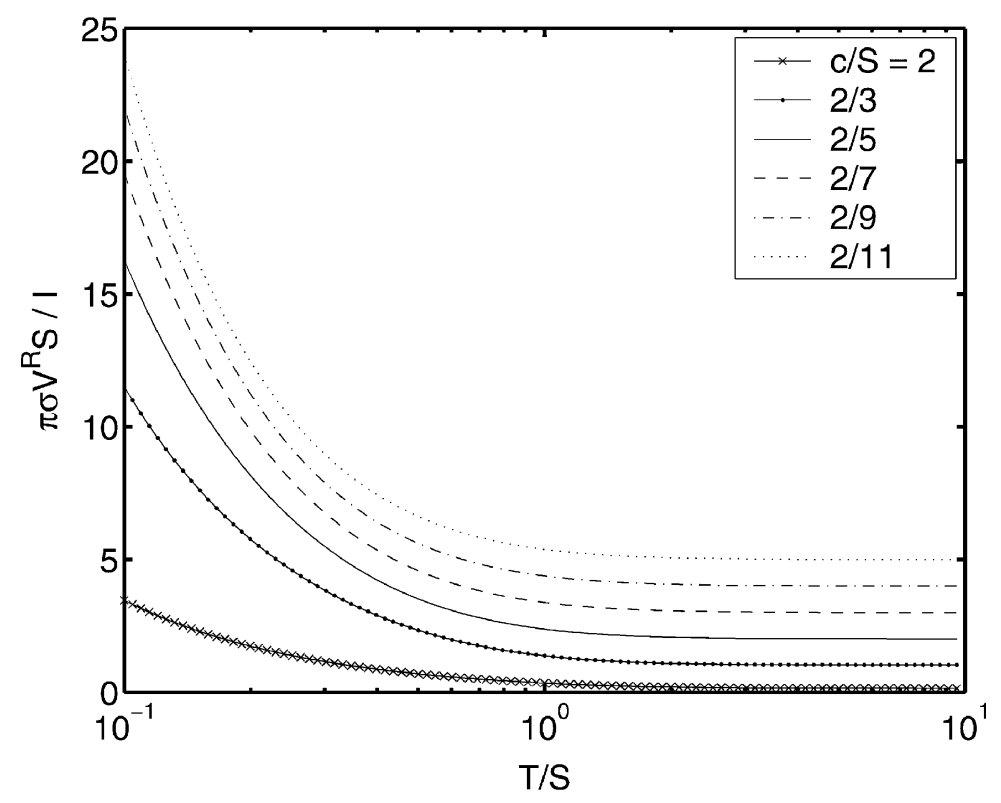

FIGURE 9. Dimensionless pickup voltage, $\pi \sigma V^{R} S / I$, in the case of a rectangular probe, computed from Eq. (25) with $N=1000$, for $c / S=2,2 / 3$ (0.667), $2 / 5$ (0.400), $2 / 7$ (0.286), $2 / 9$ (0.222), and 2/11 (0.182).

3.2.1. Thick Plate Asymptote Taking the limit $T \longrightarrow \infty$ in Eq. (25) yields the result for a half-space conductor, $V_{\infty}^{R}$. All terms in the series cancel except those for $n=0$, which gives

$$
V_{\infty}^{\mathrm{R}}=\frac{I}{\pi \sigma}\left[\frac{1}{\sqrt{(2 S)^{2}+c^{2}}}-\frac{1}{C}\right]
$$

For probe points arranged in a square with side length $C$,

$$
V_{\infty}^{S}=-\frac{l}{2 \pi \sigma c}(2-\sqrt{2})
$$

3.2.2. Thin Plate Asymptote Following the procedure of Section 2.2, or by putting $p=-S$ and $q=S$ in Eq. (18), Eq. (25) may be written in the form

$$
V^{R}=-\frac{I}{2 \pi \sigma T}\left\{\ln \left[1+\left(\frac{2 S}{C}\right)^{2}\right]-N\left(\frac{\sqrt{(2 S)^{2}+c^{2}}}{2 T}\right)+N\left(\frac{C}{2 T}\right)\right\}
$$


In the thin plate regime (whose range of validity is yet to be determined),

$$
V_{\mathrm{TP}}^{\mathrm{R}}=-\frac{I}{2 \pi \sigma T} \ln \left[1+\left(\frac{2 S}{c}\right)^{2}\right]
$$

and, for square arrangement of the probe points,

$$
V_{\mathrm{TP}}^{\mathrm{S}}=-\frac{l}{2 \pi \sigma T} \ln 2
$$

3.2.3. Validity of Asymptotic Formulae In Fig. 10, curves of $\pi \sigma V^{R} S / I$ versus $T / S$ are plotted showing the half-space asymptote, Eq. (27), the thin plate asymptote, Eq. (30), and the result valid for arbitrary plate thickness, Eq. (25). In this case $c / S=2$ and the probe points are arranged on the vertices of a square, so in fact the same results can be obtained using Eqs. (28), (31), and (26). From the figure it can be seen that the thick plate asymptote is a good approximation for plates with $T \approx 6 S=3 \mathrm{c}$ or greater, whereas the thin plate asymptote works well for plates with $T \approx S=c / 2$ or less.

The percentage difference defined in Eq. (24), but now for $V_{i}^{R}$, is plotted in Figs. 11 and 12 for $i=\infty$ and TP, respectively, for various values of $c / S$.

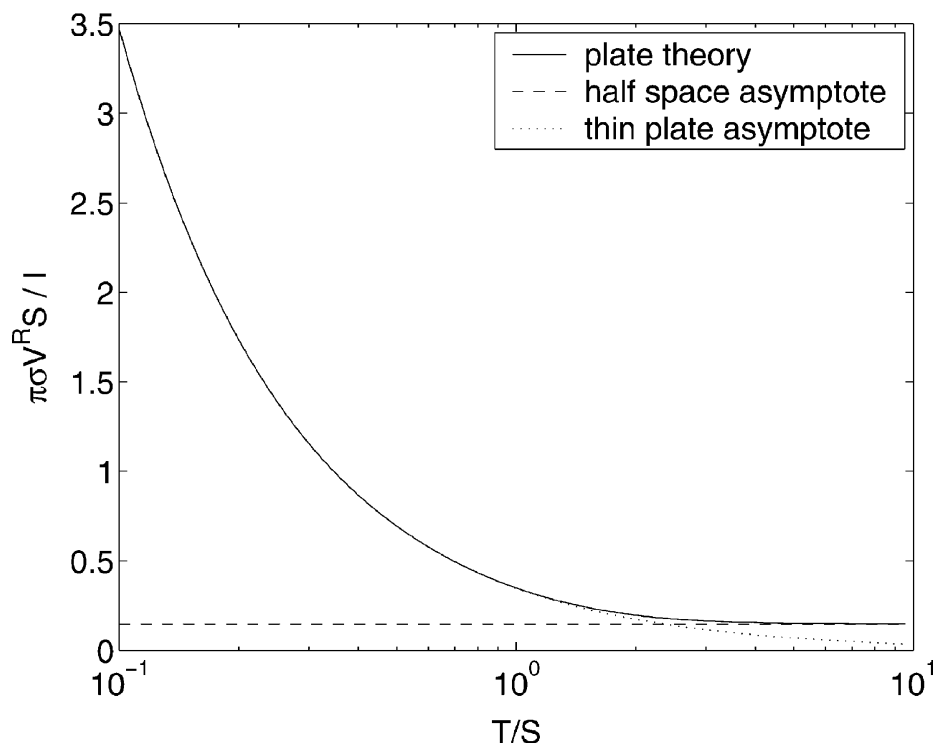

FIGURE 10. Dimensionless pickup voltage, $\pi \sigma V^{R} S / I$, in the case of a square-head probe $(c / S=2)$, plotted as a function of $T / S$. Thick and thin plate asymptotes computed from Eqs. (27) and (30), respectively, are shown. 


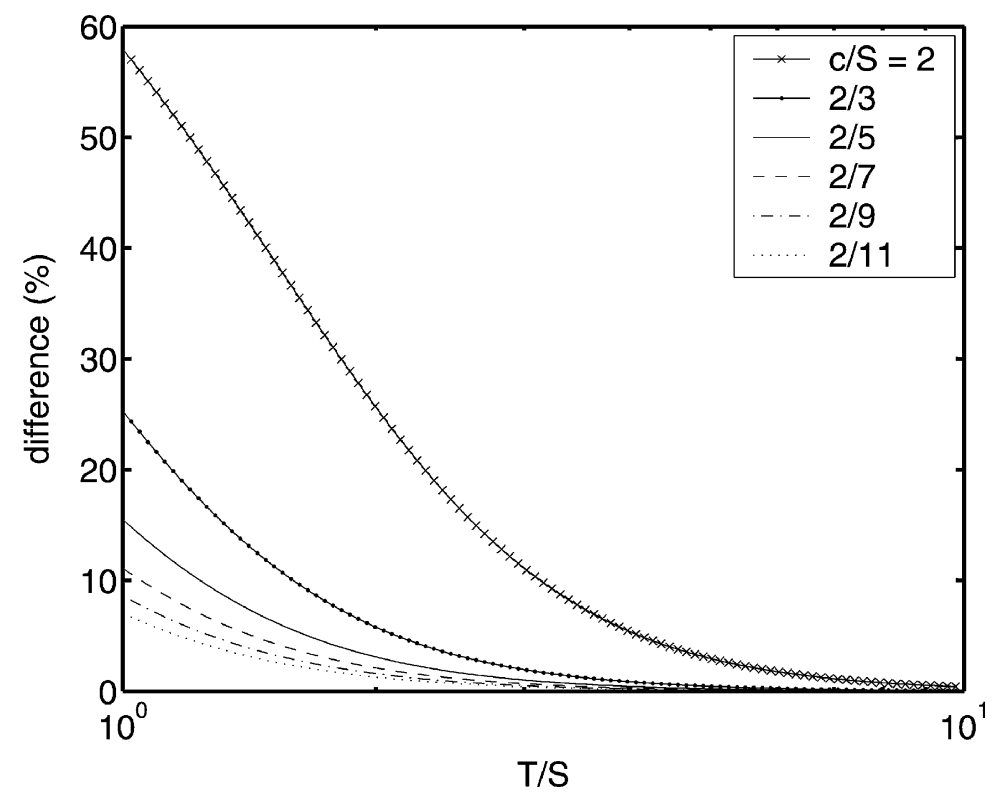

FIGURE 11. Percentage difference between pickup voltages calculated using Eq. (25) and thick plate asymptote Eq. (27) as a function of $T / S$, for various aspect ratios $c /(2 S)$ of a rectangular probe.

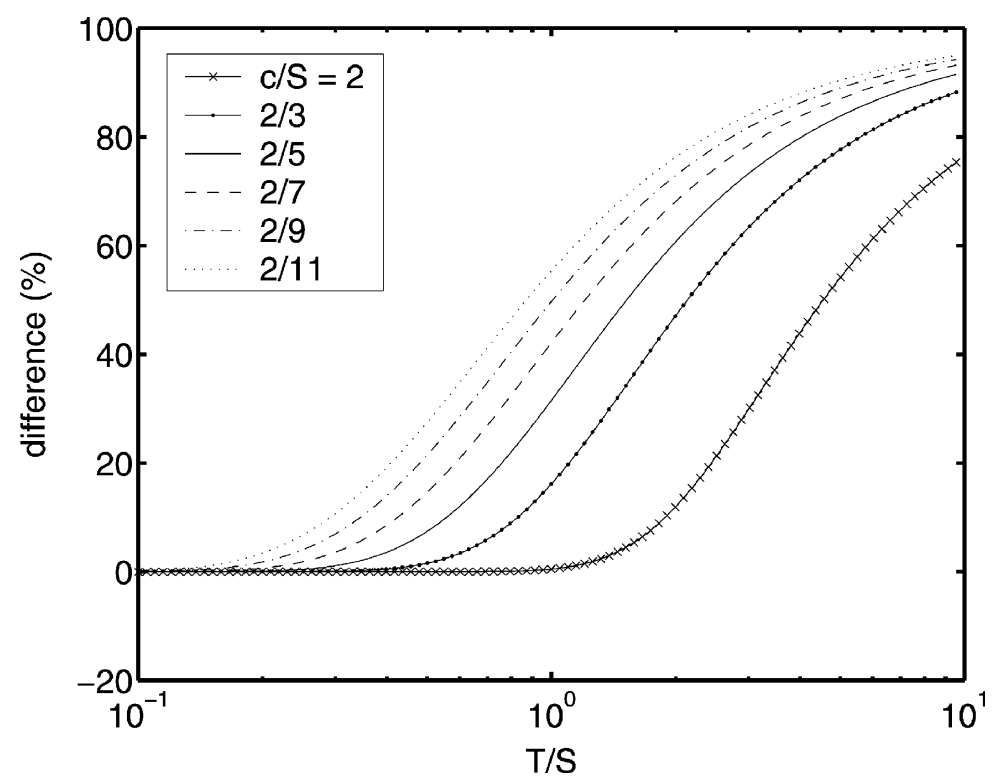

FIGURE 12. As for Fig. 11 but with thin plate asymptote Eq. (30). 


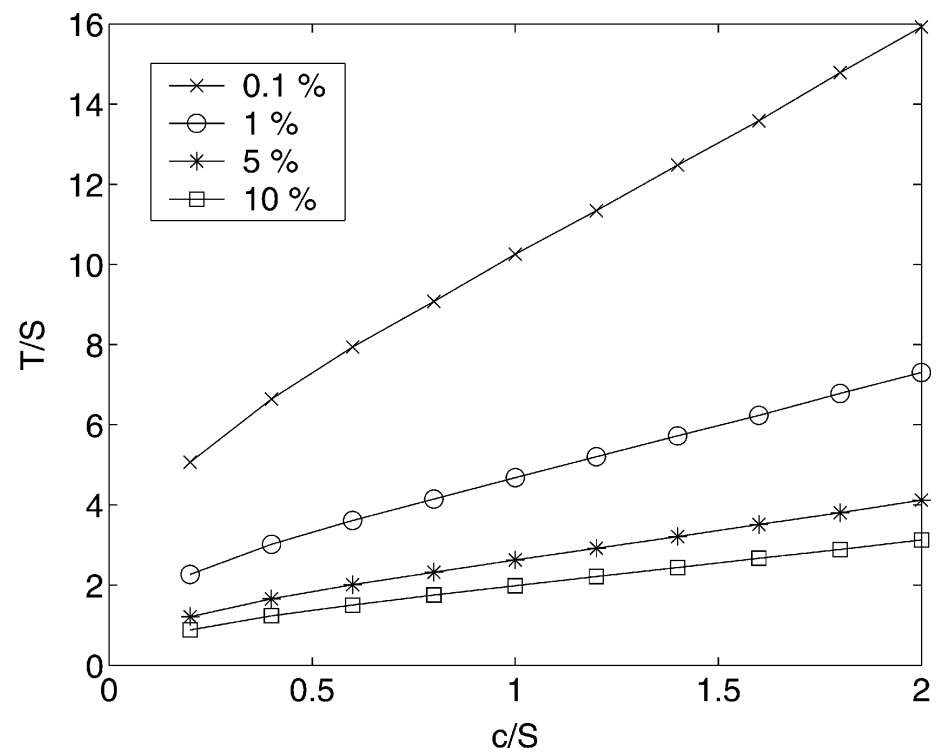

FIGURE 13. Curves of constant percentage difference between pickup voltages calculated using Eq. (25) and thick plate asymptote Eq. (27), for $T / S$ plotted versus the aspect ratio of a rectangular probe, $c / S$.

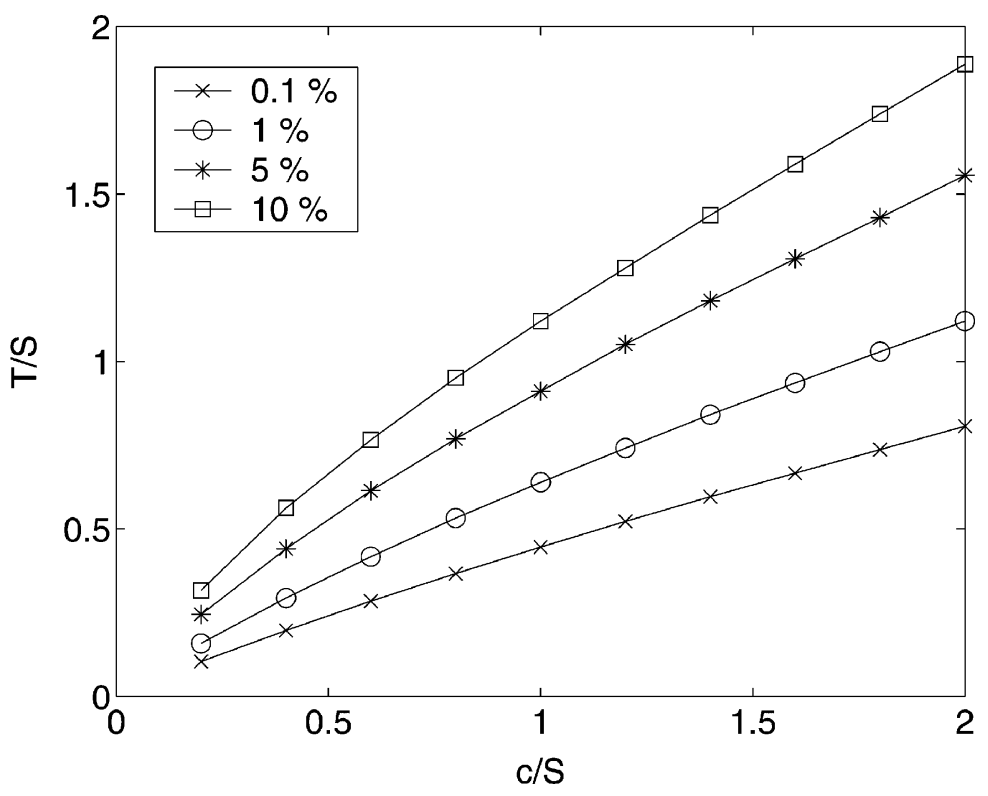

FIGURE 14. As for Fig.13 but with thin plate asymptote Eq. (30). 
It is clear, from visual inspection of the curves in Figs. 11 and 12, that the accuracy of the asymptotes for any particular plate thickness also depends on the aspect ratio of the probe, $c /(2 S)$. The thick plate asymptotic expression becomes more accurate, for a given plate thickness, as the aspect ratio decreases, that is, as $c / S$ decreases and the pickup points approach the current input points more closely. The thin plate asymptotic expression becomes more accurate in the opposite case, as the pickup points move away from the current input points ( $c / S$ increases).

In Figs. 13 and 14, curves of constant percentage difference $(0.1,1,5$, and $10 \%$ ) are plotted for $T / S$ versus $q / S$, for thick and thin plate asymptotes, respectively. These curves facilitate proper choice of probe dimensions relative to plate thickness for the level of accuracy required in utilizing asymptotic Eqs. (27) or (30).

\section{CONCLUSION}

A series solution for the potential drop between two contact points on the surface of a metal plate of arbitrary thickness is derived, for direct current injected via two further contact points. Simple analytic expressions are obtained in two asymptotic regimes; for plates thick or thin with respect to the probe dimensions. Specific formulas are given for colinear and rectangular arrangements of the probe points, with numerical assessment of the accuracy of the asymptotic expressions for various ratios of plate thickness to relevant probe dimensions. For both colinear and rectangular probes it is found that the thick plate asymptotes become more accurate as the pickup points are moved closer to the current injection points. The thin plate asymptotes become more accurate in the opposite case, as the pickup points are moved further from the current injection points. The results of this study provide a useful guide in selecting the most appropriate arrangement of probe points for measurement of the electrical conductivity of metal plates, depending on the level of accuracy required.

The expressions for the pickup voltage, here developed under the assumption of applied direct current, are also valid for applied alternating current when the frequency is below a certain value that is characteristic of the measurement system [15]. The use of alternating current allows lower measuring current to be applied to achieve a given sensitivity [1]. This has the advantage of reducing the risk of heating in the part under test and consequent changes in the material conductivity during the measurement process.

\section{ACKNOWLEDGMENTS}

This work was supported by the NSF Industry/University Cooperative Research program. The author thanks J. T. Brown for assistance with obtaining the experimental data on aluminum and titanium, Fig. 8. 


\section{REFERENCES}

1. R. K. Stanley, P. O. Moore, and P. McIntire (eds.). Nondestructive Testing Handbook, 2nd ed., vol. 9, Special Nondestructive Testing Methods. American Society for Nondestructive Testing, Columbus, $\mathrm{OH}$ (1995).

2. A. C. Lynch, A. E. Drake, and C. H. Dix. Measurement of eddy-current conductivity. IEE Proc. A 130:254 (1983).

3. G. R. Metcalfe. The use of eddy current flaw detectors with meter display for measuring the conductivity of aluminum alloy structures. British J. NDT 30:164 (1988).

4. N. Bowler and Y. Huang. Electrical conductivity measurement of metal plates using broadband eddycurrent and four-point methods. Meas. Sci. Technol. 16:2193 (2005).

5. H. Suhr and T. W. Guettinger. Error reduction in eddy current conductivity measurements. British J. NDT 35:634 (1993).

6. F. Yu and P. B. Nagy. Simple analytical approximations for eddy current profiling of the near-surface residual stress in shot-peened metals. J. Appl. Phys. 96: 1257 (2004).

7. D. K. Schroder. Semiconductor Material and Device Characterization. Wiley, New York (1998).

8. J. M. Reynolds. Introduction to Applied and Environmental Geophysics. Wiley, Chichester, UK (1997).

9. D. S. Parasnis. Principles of Applied Geophysics, 5th ed. Chapman and Hall, London (1997).

10. N. Bowler. Electric field due to alternating current injected at the surface of a metal plate. J. Appl. Phys. 96:4607 (2004).

11. N. Bowler and Y. Huang. Four-point alternating current potential drop measurements on metal plates: experiment and interpretation. Center for Nondestructive Evaluation, lowa State University, Ames, lowa 2005. Unpublished.

12. A. Uhlir, Jr. The potentials of infinite systems of sources and numerical solutions of problems in semiconductor engineering. Bell Syst. Tech. J. 34:105 (1955).

13. M. Abramowitz and I. A. Stegun (eds.). Handbook of Mathematical Functions with Formulas, Graphs, and Mathematical Tables. Dover, New York (1972).

14. S. S. Udpa and P. O. Moore (eds.). Nondestructive Testing Handbook, 3rd ed., vol. 5, Electromagnetic Testing. American Society for Nondestructive Testing, Columbus (2004).

15. N. Bowler and Y. Huang. Model-based characterization of homogeneous metal plates by four-point alternating current potential drop measurements. IEEE Trans. Mag. 41:2102 (2005). 